\title{
DEGREE OF THE DIVISOR OF SOLUTIONS OF A DIFFERENTIAL EQUATION ON A PROJECTIVE VARIETY
}

\author{
VICENTE MUÑOZ AND IGNACIO SOLS
}

December, 1998

\begin{abstract}
Using the data schemes from [5] we give a rigorous definition of algebraic differential equations on the complex projective space $\mathbb{P}^{n}$. For an algebraic subvariety $S \subseteq \mathbb{P}^{n}$, we present an explicit formula for the degree of the divisor of solutions of a differential equation on $S$ and give some examples of applications. We extend the technique and result to the real case.
\end{abstract}

\section{INTRODUCTION}

We deal with the problem of finding the degree of the divisor of solutions of a differential equation on a projective variety, which was studied by Halphen in [6] for differential equations on plane curves and on hypersurfaces of $\mathbb{P}^{n}$. With the notion of infinitesimal data in $\mathbb{P}^{n}$, introduced in the plane by A. Collino [4], used by S. Colley and G. Kennedy in [2] [3], and generalised to higher dimensions in [5], we solve this problem for algebraic subvarieties of $\mathbb{P}^{n}$ (the method of Halphen seems to generalise only to complete intersections). The plane data of Collino have been rediscovered by Mohamed Belghiti [1] and applied to give a modern proof of the Halphen formula for plane curves, including some explicit calculation of the involved invariants of the equation that Halphen was able to obtain in this case.

Let $\mathbb{P}^{n}$ the complex projective space of dimension $n$ (although we can work over any algebraically closed field $K$ of zero characteristic). Associated to a set of coordinates $x_{1}, \ldots, x_{k}, y_{1}, \ldots, y_{n-k}$ on an affine open set $U^{0}=\mathbb{C}^{n} \subseteq \mathbb{P}^{n}$, we construct inductively an open set $U^{r}$ of the data scheme $D_{k}^{r} \mathbb{P}^{n}$, where the partial derivatives of the $y_{j}$ with respect to the $x_{i}$ are understood as the canonical coordinates of $U^{r}$. In this context, a differential equation

$$
f\left(x_{i}, y_{j}, \frac{\partial y_{j}}{\partial x_{i}}, \frac{\partial^{2} y_{j}}{\partial x_{i_{2}} \partial x_{i_{1}}}, \cdots, \frac{\partial^{r} y_{j}}{\partial x_{i_{r}} \cdots \partial x_{i_{1}}}\right)=0,
$$

is understood as a algebraic equation on the open set $U^{r}$. The data satisfying the differential equation form a divisor $D_{k}^{r} \mathbb{P}^{n}(f)$ on $D_{k}^{r} \mathbb{P}^{n}$ defined as the closure of the solutions to $f=0$.

Mathematics Subject Classification. Primary: 14N10. Secondary: 14D20, 14M15, 14P05. 
For the differential equation $f$ there are naturally well defined enumerative invariants $\gamma_{s}^{f}$, $0 \leq s \leq r$.

Let $S \subseteq \mathbb{P}^{n}$ be an algebraic subvariety of dimension $k$, then $S(f)$ will be the locus of the solutions of the differential equation $f=0$ on $S$. The main result of the paper (theorem 13) gives a closed formula for $\operatorname{deg} S(f)$ (assuming $S(f)$ is a proper subset of $S$ ), expressed as the scalar product between the $\gamma_{s}^{f}$ and the cuspidal numbers $\gamma_{S}^{s}$ of $S$. This reduces to a simple formula $\operatorname{deg} S(f)=\gamma_{0}^{f} \gamma_{S}^{0}+\gamma_{1}^{f} \gamma_{S}^{1}$ when $S$ is smooth (or normal), where $\gamma_{S}^{0}$ and $\gamma_{S}^{1}$ are the degree and class of $S$ respectively, and $\gamma_{0}^{f}$ and $\gamma_{f}^{1}$ can be computed directly, either with the use of proposition 7 or remark 9 (when $f$ is distinguished in suitable variables, which is the general case) or by using the formula $\operatorname{deg} S(f)=\gamma_{0}^{f} \gamma_{S}^{0}+\gamma_{1}^{f} \gamma_{S}^{1}$ applied to a smooth quadric and a smooth cubic subvarieties $S \subseteq \mathbb{P}^{n}$, and solving the system of linear equations thus obtained.

The use of theorem 13 is two-fold. On the one hand it gives the degree of $S(f)$ if we know enough information from $f$ and $S$. This can be used to understand geometric properties of $S$ as these are usually described by a suitable differential equation (e.g. flexes of plain curves, parabolic points). On the other hand the explicit computations of $\operatorname{deg} S(f)$ in particular cases may lead to finding the invariants $\gamma_{s}^{f}$ of a differential equation $f$, and the cuspidal numbers $\gamma_{S}^{s}$ of a subvariety $S$. Section 4 is devoted to examples in which these two applications of theorem 13 are worked out. In particular, we find the degree of the divisor of parabolic points of a subvariety of $\mathbb{P}^{n}$.

Finally in the last section we extend the results for the real projective space $\mathbb{P}_{\mathbb{R}}^{n}$, by studying the behaviour of the constructions involved via the conjugation involution. This is merely an introduction and not a thorough analysis of the use of data varieties in the real case. The formula thus obtained in theorem 23 is analogue to the one in the complex case, but we have to restrict ourselves to coefficients in $\mathbb{Z} / 2 \mathbb{Z}$. We end up with a simple application on the number of umbilical points of a real surface in $\mathbb{P}_{\mathbb{R}}^{3}$.

\section{Differential equations on projective space}

To make precise sense of a differential equation in the projective space $\mathbb{P}^{n}$, we need to recall from [5] the definition of the smooth compact moduli $D_{k}^{r} \mathbb{P}^{n}$ of infinitesimal data of dimension $k$ at order $r$ in $\mathbb{P}^{n}$.

Let $Z$ be a scheme smooth over $\mathbb{C}$. We define schemes $D_{k}^{r} Z$ together with embeddings and projections

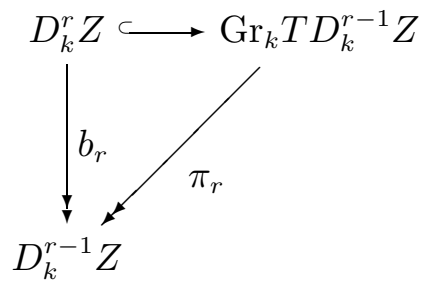


inductively, as follows. We take $D_{k}^{0} Z=Z, D_{k}^{1} Z=\mathrm{Gr}_{k} T Z$ and if $r \geq 2$ and

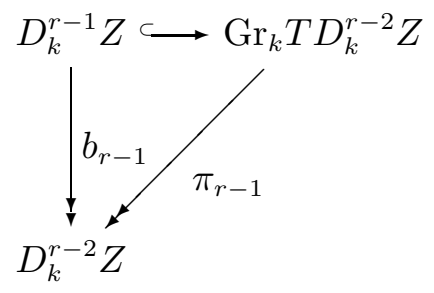

is already defined then $D_{k}^{r} Z \subseteq \operatorname{Gr}_{k} T D_{k}^{r-1} Z$ is the Grassmannian $\operatorname{Gr}_{k} \mathcal{F}_{r-1}$ of the (Semple) bundle $\mathcal{F}_{r-1} \subseteq T D_{k}^{r-1} Z$ obtained as pull-back

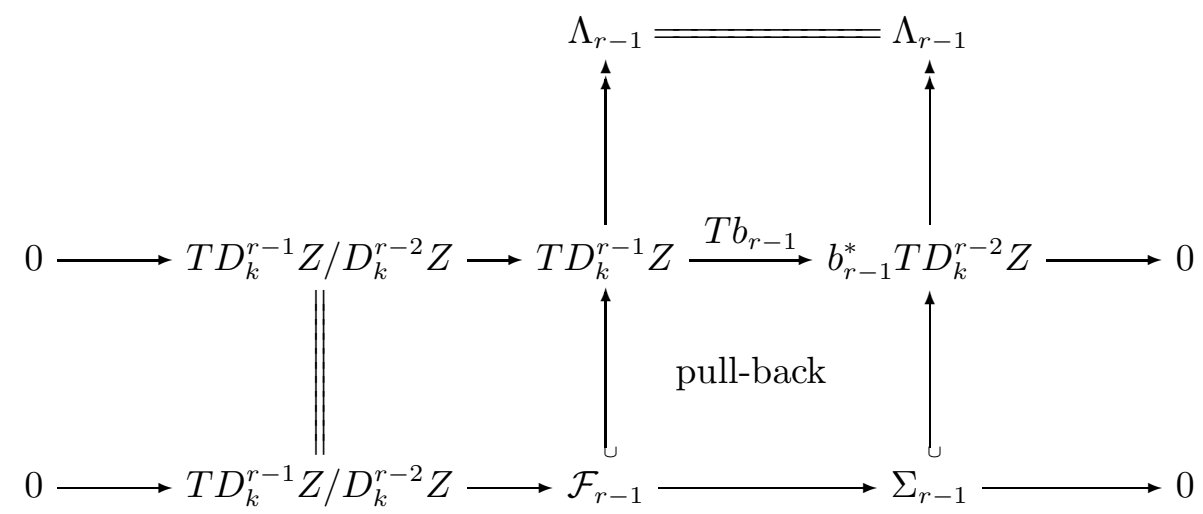

where the right hand sequence is the restriction to $D_{k}^{r-1} Z$ of the universal sequence on $\mathrm{Gr}_{k} T D_{k}^{r-2} Z$.

On each $D_{k}^{r} Z=\mathrm{Gr}_{k} \mathcal{F}_{r-1}$ there is a natural divisor $C_{k}^{r} Z$, namely the Schubert special cycle of $k$-planes of $\mathcal{F}_{r-1}$ meeting $T D_{k}^{r-1} Z / D_{k}^{r-2} Z$, whose elements are called cuspidal. The elements in the complement are called non-cuspidal.

If $Z^{\prime}$ is a subvariety of $Z$ of dimension $k$ and $Z_{\mathrm{reg}}^{\prime}$ is the open set of smooth points, then

$$
Z_{\mathrm{reg}}^{\prime} \cong D_{k}^{r} Z_{\mathrm{reg}}^{\prime} \subseteq D_{k}^{r} Z
$$

by the obvious functoriality of our construction. We then define $D_{k}^{r} Z^{\prime} \subseteq D_{k}^{r} Z$ as the closure of this subset.

From now on $Z=\mathbb{P}^{n}=\mathbb{P}(V)$ and $1 \leq k \leq n-1$. Fix a trivialisation $V \cong \mathbb{C}^{n+1}$ or projective reference, with corresponding hyperplane $C^{0}$ at infinity and affine part $U^{0}=$ $\mathbb{P}^{n}-C^{0}=\mathbb{C}^{n}=\mathbb{C}^{k} \times \mathbb{C}^{n-k}$, with affine coordinates which we name

$$
x_{1}, \ldots, x_{i}, \ldots, x_{k}, y_{1}, \ldots, y_{j}, \ldots, y_{n-k} .
$$

In order to make sense of a differential equation, we need to introduce formal partial derivative symbols of the variables $y_{j}$ with respect to the variables $x_{i}$. For any $s \geq 1,1 \leq j \leq n-k$, and $1 \leq i_{1}, \ldots, i_{s} \leq k$ we introduce

$$
y_{i_{1}, \ldots, i_{s}}^{j}=\frac{\partial^{s} y_{j}}{\partial x_{i_{s}} \cdots \partial x_{i_{1}}} .
$$


At the present stage these are to be understood merely as symbols. Later we will identify them as coordinates of an affine open set of the data scheme $D_{k}^{r} \mathbb{P}^{n}$. Note that we have introduced non-commuting derivative symbols (see remark 5 below).

On each point $P \in U^{0}$ we consider the $k$-space $X(P)=P+\mathbb{C}^{k} \subseteq T U^{0}$ and the $(n-k)$ space $Y(P)=P+\mathbb{C}^{n-k} \subseteq T U^{0}$ providing subbundles $X, Y$ of $T U^{0}=X \oplus Y$, all of them trivial. The complement $U^{1}$ in $D_{k}^{1} U^{0}=\mathrm{Gr}_{k} T U^{0}$ of the special Schubert cycle of $k$-subbundles of $T U^{0}$ meeting $Y$ is thus

$$
U^{1}=\operatorname{Hom}(X, Y)=U^{0} \times \operatorname{Hom}\left(\mathbb{C}^{k}, \mathbb{C}^{n-k}\right)=U^{0} \times V^{1},
$$

where $V^{1}=\operatorname{Hom}\left(\mathbb{C}^{k}, \mathbb{C}^{n-k}\right)$ is a cartesian power of $\mathbb{C}$ with coordinates $y_{i}^{j}$ (standing for $\frac{\partial y_{j}}{\partial x_{i}}$ ). On $U^{1}$, the lifted $k$-subbundle $X_{U^{1}} \subseteq\left(T U^{0}\right)_{U^{1}}$ provides a split of the universal sequence

$$
0 \rightarrow \Sigma_{1} \rightarrow\left(T U^{0}\right)_{U^{1}} \rightarrow \Lambda_{1} \rightarrow 0
$$

on $U^{1}$. The universal subbundle $\Sigma_{1}$ on $U^{1}$ becomes then isomorphic to the bundle $X_{U^{1}} \cong$ $U^{1} \times \mathbb{C}^{k}$.

We shall denote $C^{1}$ the closure in $D_{k}^{1} \mathbb{P}^{n}$ of the complement of $U^{1}$ in $D_{k}^{1} U^{0}$. Also for all $r \geq 2$ we let $C^{r}=C_{k}^{r} \mathbb{P}^{n}$ be the cuspidal divisor of $D_{k}^{r} \mathbb{P}^{n}$. Denote by $C_{s}^{r}$ for all $0 \leq s \leq r$ the divisor of $D_{k}^{r} \mathbb{P}^{n}$ counterimage by

$$
b_{r, s}=b_{s+1} \circ \cdots \circ b_{r-1} \circ b_{r}: D_{k}^{r} \mathbb{P}^{n} \rightarrow D_{k}^{s} \mathbb{P}^{n}
$$

of the divisor $C^{s}$ of $D_{k}^{s} \mathbb{P}^{n}$ (in particular $C_{r}^{r}=C^{r}$ ). Denote by $U^{r}$ the open subset of $D_{k}^{r} \mathbb{P}^{n}$

$$
U^{r}=D_{k}^{r} \mathbb{P}^{n} \backslash\left(C_{0}^{r} \cup \ldots \cup C_{s}^{r} \cup \ldots \cup C_{r}^{r}\right)
$$

consisting of data which are not in $C_{0}^{r}$ (i.e. which are "finite"), are not in $C_{1}^{r}$ (i.e. which are nowhere "vertical") and are not in $C_{s}^{r}$ for any $s \geq 2$ (i.e. which are "nonsingular"). It is important to note that $C_{s}^{r}$ is intrinsically defined for $s \geq 2$, but is dependent on the choice of trivialisation for $s=0,1$. The data in $U^{r}$ are the data for which the symbols (3), for $s=1, \ldots, r$, are going to acquire a precise meaning.

Lemma 1. $C_{0}^{r}, C_{1}^{r}, \ldots, C_{s}^{r}, \ldots, C_{r}^{r}$ is a basis for $A^{1} D_{k}^{r} \mathbb{P}^{n}$.

Proof. We can see this by induction on $r \geq 0$. For $r=0$ it is obvious. In general, we have to show that the natural map

$$
\operatorname{Pic} D_{k}^{r-1} \mathbb{P}^{n} \times \mathbb{Z} C^{r} \rightarrow \operatorname{Pic} D_{k}^{r} \mathbb{P}^{n}=\operatorname{Pic} \operatorname{Gr}_{k}\left(\mathcal{F}_{r-1}\right)
$$

is an isomorphism. We need only to remark that for any point $P \in D_{k}^{r-1} \mathbb{P}^{n},\left(C^{r}\right)_{P}$ is a basis of the Picard group of the Grassmannian $\operatorname{Gr}_{k}\left(\left(\mathcal{F}_{r-1}\right)_{P}\right)$. Surjectivity of $(6)$ now follows easily from [7, Ex. III.12.4] and injectivity by restricting to a fibre.

Alternatively, the lemma follows from the explicit descriptions given below. The complement of the union of all $C_{s}^{r}$ is a cartesian power of $\mathbb{C}$ (proposition 2) and $C_{s}^{r}$ are all irreducible, therefore they generate $A^{1} D_{k}^{r} \mathbb{P}^{n}$. On the other hand, by the proof of step 2 of proposition 7 , they are linearly independent. So they form a basis for $A^{1} D_{k}^{r} \mathbb{P}^{n}$. 
Proposition 2. The given trivialisation (2) of $U^{0}$ induces trivialisations

$$
U^{r} \cong U^{r-1} \times \operatorname{Hom}\left(\left(\mathbb{C}^{k}\right)^{\otimes r}, \mathbb{C}^{n-k}\right)
$$

of $U^{r}$ with cartesian powers of $\mathbb{C}$. The coordinates of $U^{r}$ are given by (2) and (3), $1 \leq s \leq r$.

Proof. Starting from the trivialisation of $U^{0}$, we get an induced trivialisation (4) of $U^{1}$. Suppose we have already trivialisations $U^{s}=U^{s-1} \times V^{s}$ with $V^{s} \cong \operatorname{Hom}\left(\left(\mathbb{C}^{k}\right)^{\otimes s}, \mathbb{C}^{n-k}\right)$, which is isomorphic to a cartesian power of $\mathbb{C}$, for $1 \leq s \leq r$. Then $\left(\Sigma_{r}\right)_{U^{r}} \cong\left(\Sigma_{r-1}\right)_{U^{r}} \cong$ $\cdots \cong\left(\Sigma_{1}\right)_{U^{r}} \cong X_{U^{r}} \cong U^{r} \times \mathbb{C}^{k}$. The Semple sequence on $U^{r}$ is split by

$$
\mathcal{F}_{r} \hookrightarrow T U^{r} \rightarrow T U^{r} / U^{r-1}
$$

for all $r$, so that $\mathcal{F}_{r}=\Sigma_{r} \oplus T U^{r} / U^{r-1}$. Consequently,

$$
U^{r+1} \cong \operatorname{Hom}\left(\left(\Sigma_{r}\right)_{U^{r}}, T U^{r} / U^{r-1}\right)=U^{r} \times \operatorname{Hom}\left(\mathbb{C}^{k}, V^{r}\right),
$$

i.e. $U^{r+1} \cong U^{r} \times V^{r+1}$ with $V^{r+1} \cong \operatorname{Hom}\left(\left(\mathbb{C}^{k}\right)^{\otimes(r+1)}, \mathbb{C}^{n-k}\right)$, as required.

As for naming the coordinates corresponding to this chart $U^{r}$ of $D_{k}^{r} \mathbb{P}^{n}$, these are given by (2) for $U^{0}, y_{i}^{j}$ for $V^{1}$, and in general, assuming $y_{i_{1}, \ldots, i_{r}}^{j}$ are coordinates for $V^{r}$, then the space $V^{r+1} \cong \operatorname{Hom}\left(\mathbb{C}^{k}, V^{r}\right)$ is described by coordinates which we denote $\frac{\partial y_{i_{1}}^{j} \ldots, i_{r}}{\partial x_{i_{r+1}}}$, i.e. $y_{i_{1}, \ldots, i_{r}, i_{r+1}}^{j}$.

For later use, there is a zero section $0_{r}: U^{r-1} \rightarrow U^{r}$ of $b_{r}: U^{r} \rightarrow U^{r-1}$ and composing, a zero section $0_{s, r}: U^{s} \rightarrow U^{r}$ of $b_{r, s}$, for all $s \leq r$. Now we are in the position of defining differential equations on $\mathbb{P}^{n}$.

Definition 3. A differential equation on $\mathbb{P}^{n}$ relative to a reference $x_{i}, y_{j}$ is a non-zero algebraic equation

$$
f\left(x_{i}, y_{j}, y_{i}^{j}, y_{i_{1}, i_{2}}^{j}, \ldots, y_{i_{1}, \ldots, i_{r}}^{j}\right)=0
$$

on $U^{r}$, for some $r \geq 1$.

Let $U^{r}(f)$ be the divisor of $U^{r}$ defined by the differential equation $f$, and let $H_{f}=$ $D_{k}^{r} \mathbb{P}^{n}(f)$ denote its closure in $D_{k}^{r} \mathbb{P}^{n}$, i.e. the data satisfying the differential equation. In this way, any differential equation $f$ gives a hypersurface $H_{f}$ in $D_{k}^{r} \mathbb{P}^{n}$ not containing any of $C_{2}^{r}, \ldots, C_{r}^{r}$. Conversely, given a hypersurface $H$ in $D_{k}^{r} \mathbb{P}^{n}$ not containing any of $C_{2}^{r}, \ldots, C_{r}^{r}$, we can find a suitable reference (2) such that $H$ does not contain $C_{0}^{r}$ and $C_{1}^{r}$, thus being defined as $D_{k}^{r} \mathbb{P}^{n}(f)$ for a suitable polynomial $f$ on $U^{r}$. So we can give a more intrinsic definition

Definition 4. A differential equation on $\mathbb{P}^{n}$ is a hypersurface $H \subseteq D_{k}^{r} \mathbb{P}^{n}$ not containing any of $C_{2}^{r}, \ldots, C_{r}^{r}$.

This second viewpoint allows us to forget coordinates. Nonetheless, in practice, we need to work with coordinates. Whenever we say that $f$ is a differential equation on the projective space, we shall understand that there is a projective reference implicit. There are two caveats. First, if we change the reference, the polynomial $f$ will change in general. 
Second, there might be some forbidden references that we cannot choose (namely, those in which $H_{f}$ contains either of $C_{0}^{r}, C_{1}^{r}$ ).

Remark 5. The formal partial derivative symbols we have defined are non-commuting. This simply means that, for instance, $y_{i_{1}, i_{2}}^{j}$ and $y_{i_{2}, i_{1}}^{j}\left(i_{1} \neq i_{2}\right)$ are independent coordinates. We can solve this difficulty by restricting to the subset of $U^{r}$ given by the equations $y_{i_{1}, \ldots, i_{s}}^{j}=$ $y_{\sigma\left(i_{1}\right), \ldots, \sigma\left(i_{s}\right)}^{j}, 1 \leq j \leq n-k, 1 \leq s \leq r, \sigma$ any permutation of indices, and considering the closure of such subset in $D_{k}^{r} \mathbb{P}^{n}$.

More intrinsically, this subvariety of symmetric data $S_{k}^{r} Z \subseteq D_{k}^{r} Z$ has been extracted in [5], for any smooth scheme $Z$. In our case, dealing with $D_{k}^{r} Z$ is simpler and will suffice.

Associated to a differential equation we have naturally defined enumerative invariants, thanks to lemma 1.

Definition 6. For a differential equation $f$ on $\mathbb{P}^{n}$, define $\gamma_{0}^{f}, \ldots, \gamma_{r}^{f} \in \mathbb{Z}$ by

$$
\left[D_{k}^{r} \mathbb{P}^{n}(f)\right]=\gamma_{0}^{f} C_{0}^{r}+\cdots+\gamma_{r}^{f} C_{r}^{r}
$$

in $A^{1} D_{k}^{r} \mathbb{P}^{n}$.

It is important to note that $\gamma_{s}^{f}$ do not depend on the trivialisation, as the divisor classes of $C_{0}^{r}$ and $C_{1}^{r}$ are independent of the trivialisation as well. The following proposition allows us to compute some of $\gamma_{s}^{f}$ in the general case.

Proposition 7. Let $f$ be a differential equation on $\mathbb{P}^{n}$ relative to a reference $x_{i}, y_{j}$.

- Suppose $f$ is distinguished in the variable $y_{1,(r), 1}^{1}$ (i.e. for $d=\operatorname{deg}(f)$ the monomial $\left(y_{1,(r), 1}^{1}\right)^{d}$ appears in $f$ with nonzero coefficient $)$, then the leading $\gamma_{r}^{f}$ is

$$
\gamma_{r}^{f}=\operatorname{deg} f\left(0, \ldots, y_{1,(r), 1}^{1}, \ldots, 0\right) .
$$

- Suppose $f$ is distinguished in the variable $y_{1}^{1}$, then

$$
\gamma_{1}^{f}=\operatorname{deg} f\left(0, \ldots, y_{1}^{1}, \ldots, 0\right) .
$$

- Suppose $f$ is distinguished in the variable $x_{1}$, then

$$
\gamma_{0}^{f}=\operatorname{deg} f\left(x_{1}, \ldots, 0\right) .
$$

Proof. Step 1. We need first to exhibit a suitable basis $c_{0}^{r}, \ldots, c_{s}^{r}, \ldots, c_{r}^{r}$ of $A_{1} D_{k}^{r} \mathbb{P}^{n}$ (we name equally closed subsets, their associated cycles, and their rational classes). These will be the closure in $D_{k}^{r} \mathbb{P}^{n}$ of the one dimensional subschemes ${ }_{c}^{r} \subseteq U^{r}$ defined by the vanishing of all coordinates but $y_{1, \ldots, 1}^{1}$ (if $s=0$, this is the coordinate $x_{1}$ ). That they form a basis will be proved in Step 2 .

To give an intrinsic description of ${ }^{\circ}{ }_{s}^{r}$, we define the subspace $\mathbb{C}^{k-1} \subseteq \mathbb{C}^{k}$ in the given affine plane $\mathbb{C}^{n} \subseteq U^{0}$ as the space corresponding to coordinates $x_{2}, \ldots, x_{k}$, and the rank $(k-1)$ subbundle $\tilde{X}$ of the bundle $X$ on this plane by $\tilde{X}(P)=P+\mathbb{C}^{k-1}$, and correspondingly the trivial rank $(k-1)$-subbundle $\left(\tilde{\Sigma}_{r}\right)_{U^{r}} \cong \tilde{X}_{U^{r}}$ of $\left(\Sigma_{r}\right)_{U^{r}} \cong X_{U^{r}}$. Analogously, if $\mathbb{C} \subseteq \mathbb{C}^{n-k}$ is the $y_{1}$-axis, we define the rank 1 subbundle $\tilde{Y}$ of $Y$ by $\tilde{Y}(P)=P+\mathbb{C}$. 
Furthermore, we define inductively $\tilde{U}^{r}=\tilde{U}^{r-1} \times \mathbb{C}$ inside $U^{r}=U^{r-1} \times V^{r}$ by taking $\tilde{U}^{0}=U^{0}$

$$
\tilde{U}^{1}=\operatorname{Hom}(X / \tilde{X}, \tilde{Y}) \subseteq \operatorname{Hom}(X, Y)=U^{1},
$$

and assuming that $\tilde{U}^{r}$ is already defined, by taking

$$
\tilde{U}^{r+1}=\operatorname{Hom}\left(\left(\Sigma_{r}\right)_{U^{r}} /\left(\tilde{\Sigma}_{r}\right)_{U^{r}}, T \tilde{U}^{r} / U^{r-1}\right)=\operatorname{Hom}\left(U^{r} \times \mathbb{C}, \tilde{U}^{r} \times \mathbb{C}\right)=\tilde{U}^{r} \times \mathbb{C}
$$

inside $U^{r+1}=\operatorname{Hom}\left(\left(\Sigma_{r}\right)_{U^{r}}, T U^{r} / U^{r-1}\right)$.

Let 0 be the origin of $\mathbb{C}^{n}$, and let $0_{s-1}=0_{0, s-1}(0)$ be the origin of $U^{s-1}$. Note that $0_{s-1} \in \tilde{U}_{s-1}$. Define $\stackrel{\circ}{c}^{0}=0+\mathbb{C} \subseteq U^{0}$, where $\mathbb{C} \subseteq \mathbb{C}^{k}$ is the $x_{1}$-axis. For $s \geq 1$, let $\stackrel{\circ}{c}^{s} \subseteq U^{s}$ be the 1-dimensional subvariety $\tilde{U}^{s} \cap b_{s}^{-1}\left(0_{s-1}\right)$ and $c^{s}$ the closure of ${ }^{s}$ in $D_{k}^{s} \mathbb{P}^{n}$. For $0 \leq s \leq r$, the above $\stackrel{\circ}{c} r_{s}^{r} \subseteq U^{r}$ is just $0_{s, r}\left(\stackrel{\circ}{c}^{s}\right)$, thus $b_{r, s}\left(\stackrel{\circ}{c} r_{s}^{r}\right)=\stackrel{\circ}{c}^{s}$ and $b_{r, s}\left(c_{s}^{r}\right)=c^{s}$.

Step 2. Now we want to relate the elements $c_{0}^{r}, \ldots, c_{s}^{r}, \ldots, c_{r}^{r}$ of $A_{1} D_{k}^{r} \mathbb{P}^{n}$ with the elements $C_{0}^{r}, \ldots, C_{s}^{r}, \ldots, C_{r}^{r}$ of $A^{1} D_{k}^{r} \mathbb{P}^{n}$. One has ${ }_{c}^{r} \cong \mathbb{C}$ and therefore $c_{s}^{r} \cap\left(C_{0}^{r} \cup \ldots \cup C_{r}^{r}\right)=$ $c_{s}^{r} \backslash U^{r}=c_{s}^{r} \backslash{ }_{c}^{r} c_{s}^{r} \cong \mathbb{P} \backslash \mathbb{C}$ consists, set-theoretically, of just one point, which we want now to show not to be in any $C_{t}^{r}$ for $t<s$ and that this point is in fact the schematic intersection $c_{s}^{r} \cap C_{s}^{r}$, i.e. that $c_{s}^{r} \cdot C_{s}^{r}=1$, as rational classes. On the one hand, this gives an alternative proof that the $C_{s}^{r}$ are linearly independent in lemma 1. On the other hand, this proves that $c_{s}^{r}$ are a basis for $A_{1} D_{k}^{r} \mathbb{P}^{n}$, since the intersection matrix $\left(C_{t}^{r} \cdot c_{s}^{r}\right)$ is lower-triangular.

Let $0 \leq t<s \leq r$. Since $C_{t}^{r}=b_{r, t}^{-1}\left(C^{t}\right)$, in order to show that $c_{s}^{r} \cap C_{t}^{r}=\varnothing$ it is enough to show that $b_{r, t}\left(c_{s}^{r}\right) \cap C^{t}$ is empty. By construction, $b_{r, t}\left(c_{s}^{r}\right)=0 \in U^{t}$, since $t<s$, thus $b_{r, t}\left(c_{s}^{r}\right)=0$ is disjoint with $C^{t}$. This proves that, as rational classes, $c_{s}^{r} \cdot C_{t}^{r}=0$.

Again, since $C_{s}^{r}=b_{r, s}^{-1}\left(C^{s}\right)$, in order to show that $c_{s}^{r} \cdot C_{s}^{r}=1$, it is enough to show that $b_{r, s}\left(c_{s}^{r}\right) \cdot C^{s}=c^{s} \cdot C^{s}=1$. For $s=0$ this is true since $c^{0} \subseteq \mathbb{P}^{n}$ is a line and $C^{0}$ is the hyperplane. For $s \geq 1$, we note that since $c^{s} \subseteq b_{s}^{-1}\left(0_{s-1}\right)$, it is enough to show for $C_{0_{s-1}}^{s}=C^{s} \cap b_{s}^{-1}\left(0_{s-1}\right)$ that

$$
c^{s} \cdot C_{0_{s-1}}^{s}=1
$$

in the Grassmannian $b_{s}^{-1}\left(0_{s-1}\right)$. Suppose now that $s \geq 2$ (the case $s=1$ is similar and is left to the reader). Then $b_{s}^{-1}\left(0_{s-1}\right)=\operatorname{Gr}_{k}\left(\left(\mathcal{F}_{s-1}\right)_{0_{s-1}}\right)$, where $\left(\mathcal{F}_{s-1}\right)_{0_{s-1}}$ is the Semple vector space

$$
\left(\mathcal{F}_{s-1}\right)_{0_{s-1}}=\left(T U^{s-1} / U^{s-2}\right)_{0_{s-1}} \oplus\left(\Sigma_{s-1}\right)_{0_{s-1}} .
$$

In this Grassmannian, $C_{0_{s-1}}^{s}$ is the Schubert cycle of $k$-spaces meeting $\left(T U^{s-1} / U^{s-2}\right)_{0_{s-1}}$, i.e. the base of its Picard group $A^{1}$. On the other hand, the 1-dimensional subvariety

$$
\stackrel{\circ}{c}^{s}=\operatorname{Hom}\left(\left(\Sigma_{s-1}\right)_{0_{s-1}} /\left(\tilde{\Sigma}_{s-1}\right)_{0_{s-1}},\left(T \tilde{U}^{s-1} / U^{s-2}\right)_{0_{s-1}}\right)
$$

of the open subset $\operatorname{Hom}\left(\left(\Sigma_{s-1}\right)_{0_{s-1}},\left(T U^{s-1} / U^{s-2}\right)_{0_{s-1}}\right)$ of $\operatorname{Gr}_{k}\left(\left(\mathcal{F}_{s-1}\right)_{0_{s-1}}\right)$ is an open subset of the Grassmannian of $k$-subspaces of the $(k+1)$-subspace $\left(T \tilde{U}^{s-1} / U^{s-2}\right)_{0_{s-1}} \oplus\left(\Sigma_{s-1}\right)_{0_{s-1}} \subseteq$ $\left(\mathcal{F}_{s-1}\right)_{0_{s-1}}$ which contain the $(k-1)$-subspace $\left(\tilde{\Sigma}_{s-1}\right)_{0_{s-1}} \subseteq\left(\Sigma_{s-1}\right)_{0_{s-1}} \subseteq\left(\mathcal{F}_{s-1}\right)_{0_{s-1}}$. Thus $c^{s}$ is the Grassmannian of such $k$-spaces, i.e. the Schubert cycle base of $A_{1}$ dual to the base $C_{0_{s-1}}^{s}$ of $A^{1}$, thus $c^{s} \cdot C_{0_{s-1}}^{s}=1$. 
Step 3. Now we can find the coefficient $\gamma_{r}^{f}$ in the expression of definition 6. By the above, this is

$$
\gamma_{r}^{f}=\left[D_{k}^{r} \mathbb{P}^{n}(f)\right] \cdot c_{r}^{r}=\text { length } U^{r}(f) \cap \stackrel{\circ}{c_{r}^{r}}=\operatorname{deg} f\left(0, \ldots, y_{1,(r), 1}^{1}, \ldots, 0\right) .
$$

Indeed, the second equality is due to

$$
\left(D_{k}^{r} \mathbb{P}^{n}(f) \backslash U^{r}(f)\right) \cap c_{r}^{r}=D_{k}^{r} \mathbb{P}^{n}(f) \cap\left(C_{0}^{r} \cup \cdots \cup C_{r}^{r}\right) \cap c_{r}^{r}=\varnothing
$$

since $\left(C_{0}^{r} \cup \cdots \cup C_{r}^{r}\right) \cap c_{r}^{r}$ is schematically one point, say the point $(0, \ldots, \infty, \ldots, 0)$ at infinity of $\stackrel{\circ}{r}_{r}^{r}=\left\{\left(0, \ldots, y_{1,(r), 1}^{1}, \ldots, 0\right)\right\} \cong \mathbb{C}$, and this point is not in $D_{k}^{r} \mathbb{P}^{n}(f)$, i.e.

$$
0 \neq f(0, \ldots, \infty, \ldots, 0)=\lim _{y_{1 \ldots 1}^{1} \rightarrow \infty} f\left(0, \ldots, y_{1, \ldots, p, 1}^{1}, \ldots, 0\right)
$$

because $f$ is distinguished in the variable $y_{1,(r), 1}^{1}$. This finishes the proof of the first item in the statement of the proposition.

Step 4. To get the coefficient $\gamma_{1}^{f}$, we prove first that $c_{1}^{r} \cdot C_{s}^{r}=0$ for $s>1$. For this we consider the (flat) family of subvarieties $\left\{S_{\lambda}\right\}_{\lambda \in \mathbb{P}^{1}}$ of $\mathbb{P}^{n}$ such that for $\lambda \in \mathbb{C}, S_{\lambda}$ is given by the equations $y_{1}-\lambda x_{1}=0, y_{2}=0, \ldots, y_{n-k}=0$, and $S_{\infty}$ has equations $x_{1}=0, y_{2}=0$, $\ldots, y_{n-k}=0$. Clearly the points of ${ }^{\circ}{ }_{1}^{r}$ are parametrized by $D_{k}^{r} S_{\lambda} \cap b_{r, 0}^{-1}(0), \lambda \in \mathbb{C}$. Therefore $c_{1}^{r} \backslash c_{1}^{r}$ is the point $D_{k}^{r} S_{\infty} \cap b_{r, 0}^{-1}(0)$. But $S_{\infty}$ is smooth, so $D_{k}^{r} S_{\infty}$ is disjoint from $C_{s}^{r}=0$ for $s>1$ (see remark 11). This proves that $c_{1}^{r} \cdot C_{s}^{r}=0$ for $s>1$.

Now $\gamma_{1}^{f}=\left[D_{k}^{r} \mathbb{P}^{n}(f)\right] \cdot c_{1}^{r}=\operatorname{deg} f\left(0, \ldots, y_{1}^{1}, \ldots, 0\right)$, when $f$ is distinguished in the variable $y_{1}^{1}$, arguing as in step 3 .

Step 5. To get the coefficient $\gamma_{0}^{f}$, we need to prove first that $c_{0}^{r} \cdot C_{s}^{r}=0$ for $s>0$. This time we fix the subvariety $S$ with equations $y_{1}=0, y_{2}=0, \ldots, y_{n-k}=0$. Recall $\stackrel{\circ}{c}^{0}=0+\mathbb{C} \subseteq U^{0}$, where $\mathbb{C} \subseteq \mathbb{C}^{k}$ is the $x_{1}$-axis and $c^{0}$ is its closure in $\mathbb{P}^{n}$. The points of $c_{0}^{r}$ are parametrized by $D_{k}^{r} S \cap b_{r, 0}^{-1}(\lambda), \lambda \in c^{0} \subseteq \mathbb{P}^{n}$. Now $D_{k}^{r} S$ is disjoint from $C_{s}^{r}$, for $s \geq 1$, so $c_{0}^{r} \cdot C_{s}^{r}=0$ for $s>0$. The rest of the argument is as in steps 3 and 4 .

Remark 8. The explicit computation of the other $\gamma_{s}^{f}, 2 \leq s<r$, requires the full knowledge of the intersection matrix $c_{s}^{r} \cdot C_{t}^{r}$, which is a more delicate issue (see remark 14). Nonetheless if we are only interested in applications of differential equations on smooth varieties, $\gamma_{0}^{f}$ and $\gamma_{1}^{f}$ will suffice (see corollary 15).

Remark 9. Suppose that $f\left(x_{i}, y_{j}, y_{i}^{j}, y_{i_{1}, i_{2}}^{j}, \cdots, y_{i_{1}, \ldots, i_{r}}^{j}\right)$ is a differential equation on $\mathbb{P}^{n}$ relative to a reference $x_{i}, y_{j}$ with $d=\operatorname{deg}(f)$. Suppose that

$$
\operatorname{deg} f\left(0, \ldots, 0, y_{1,(r), 1}^{1}, \ldots, y_{k,(r), k}^{n-k}\right)=d .
$$

Then $\gamma_{r}^{f}=d$. The proof is similar to steps 2 and 3 in the proof of proposition 7 . Choose a generic line $\stackrel{\circ}{l} \subseteq V^{r}$ and identify it with $0_{r-1} \times \stackrel{\circ}{l} \subseteq U^{r}=U^{r-1} \times V^{r}$. Let $l \subseteq D_{k}^{r} \mathbb{P}^{n}$ be its closure. Then it is easy to see that $l \cdot C_{s}^{r}=0$ for $s<r$ and $l \cdot C_{r}^{r}=1$. Now

$$
\gamma_{r}^{f}=\left[D_{k}^{r} \mathbb{P}^{n}(f)\right] \cdot l=\operatorname{deg} f\left(0, \ldots, 0, y_{1, \stackrel{(r)}{1}, 1}^{1}, \ldots, y_{k,(r), k}^{n-k}\right)=d,
$$

as in step 3 in the proof of proposition 7 . 


\section{Degree of the Divisor of SOlutions}

Let $S$ be a subvariety of $\mathbb{P}^{n}$ of dimension $k$. We have defined $D_{k}^{r} S \subseteq D_{k}^{r} \mathbb{P}^{n}$ as the closure of $D_{k}^{r} S_{\text {reg }} \subseteq D_{k}^{r} \mathbb{P}^{n}$. There is a natural map

$$
D_{k}^{r} S \rightarrow S,
$$

which is an isomorphism over the smooth part. Therefore if $S$ is smooth then $S \cong D_{k}^{r} S$. In general, we have the following invariants associated to $S$

Definition 10. The cuspidal numbers $\gamma_{S}^{s}, s \geq 0$, of $S$ are defined as

- $\gamma_{S}^{0}$ is the degree of $S \subseteq \mathbb{P}^{n}$.

- $\gamma_{S}^{1}$ is the class of $S$, i.e. the degree of the divisor of $S$ consisting of the points of tangency of tangent $k$-planes to $S$ that can be drawn from a generic $(n-k-1)$-plane of $\mathbb{P}^{n}$.

- For $s \geq 2, \gamma_{S}^{s}$ is the degree of the push-forward $b_{s, 0}\left(C^{s} S\right) \subseteq \mathbb{P}^{n}$ of $C^{s} S=D_{k}^{s} S \cap C^{s} \subseteq$ $D_{k}^{s} \mathbb{P}^{n}$ under $b_{s, 0}: D_{k}^{s} \mathbb{P}^{n} \rightarrow \mathbb{P}^{n}$.

Remark 11. As remarked in [5], if $S$ is smooth then $\gamma_{S}^{s}=0$ for $s \geq 2$. Indeed, in this case, $D_{k}^{r} S$ is disjoint from the cuspidal divisors $C_{s}^{r}, s \geq 2$. Moreover if $S$ has singularities only in codimensions 2 or more (e.g. $S$ normal), the cycle $b_{s, 0}\left(C^{s} S\right) \subseteq \mathbb{P}^{n}, s \geq 2$, has dimension less or equal than $k-2$ and hence $\gamma_{S}^{s}=0$ for $s \geq 2$. If $S$ has no cuspidal singularities (for instance, if the only singularities are normal double crossings along a smooth subvariety) then $\gamma_{S}^{s}=0$ for $s \geq 2$ as well. The cuspidal numbers measure in some sense how complicated the singularities (on codimension 1) of $S$ are.

Lemma 12. For any $0 \leq s \leq r$, it is $\gamma_{S}^{s}=D_{k}^{r} S \cap C_{s}^{r} \cap H^{k-1}$, where $H=C_{0}^{r}=b_{r, 0}^{-1}(H)$ is the hyperplane in $D_{k}^{r} \mathbb{P}^{n}$.

Proof. Using that $D_{k}^{r} S \cap C_{s}^{r} \cap b_{r, 0}^{-1}\left(H^{k-1}\right)=D_{k}^{s} S \cap C^{s} \cap b_{s, 0}^{-1}\left(H^{k-1}\right)$, we reduce to the case $r=s$. Now for $r=0$ is obvious, for $r=1$ follows from the definition, and for $r \geq 2$, $D_{k}^{r} S \cap C^{r} \cap b_{r, 0}^{-1}\left(H^{k-1}\right)=C^{r} S \cdot b_{r, 0}^{-1}\left(H^{k-1}\right)=b_{r, 0}\left(C^{r} S\right) \cdot H^{k-1}=\gamma_{S}^{r}$.

Given a differential equation $f$ on $\mathbb{P}^{n}$, we define the divisor of solutions of $f$ on $S$ as follows. First, $D_{k}^{r} S(f)$ is the schematic intersection in $D_{k}^{r} \mathbb{P}^{n}$

$$
D_{k}^{r} S(f)=D_{k}^{r} S \cap D_{k}^{r} \mathbb{P}^{n}(f) .
$$

Unless $D_{k}^{r} S(f)=D_{k}^{r} S$, we have that $D_{k}^{r} S(f)$ is a divisor in $D_{k}^{r} S$. The divisor of solutions $S(f) \subset S$ of $f$ on $S$ is the push-forward of $D_{k}^{r} S(f)$ under $b_{r, 0}: D_{k}^{r} \mathbb{P}^{n} \rightarrow \mathbb{P}^{n}$, i.e. $S(f)=$ $b_{r, 0}\left(D_{k}^{r} S(f)\right)$.

Theorem 13. Let $f$ be a differential equation on $\mathbb{P}^{n}$ with $\gamma_{s}^{f}, 0 \leq s \leq r$. Let $S$ be a subvariety of $\mathbb{P}^{n}$ of dimension $k$. Suppose that $S(f)$ is a proper subset of $S$. Then the degree of $S(f)$ is

$$
\gamma_{0}^{f} \gamma_{S}^{0}+\cdots+\gamma_{s}^{f} \gamma_{S}^{s}+\cdots+\gamma_{r}^{f} \gamma_{S}^{r}
$$


Proof. The numbers $\gamma_{s}^{f}$ are defined by the condition

$$
\left[D_{k}^{r} \mathbb{P}^{n}(f)\right]=\gamma_{0}^{f} C_{0}^{r}+\cdots+\gamma_{s}^{f} C_{s}^{r}+\cdots+\gamma_{r}^{f} C_{r}^{r}
$$

in $A^{1} D_{k}^{r} \mathbb{P}^{n}$. Now the degree of $S(f)$ is $S(f) \cdot H^{k-1}$ i.e.

$$
D_{k}^{r} S(f) \cdot b_{r, 0}^{-1}\left(H^{k-1}\right)=D_{k}^{r} S \cap D_{k}^{r} \mathbb{P}^{n}(f) \cap H^{k-1}=\left[D_{k}^{r} S \cap H^{k-1}\right] \cdot\left[D_{k}^{r} \mathbb{P}^{n}(f)\right] .
$$

Lemma 12 says that the $s^{t h}$-cuspidal degree of $S$ is given as $\gamma_{S}^{s}=\left[D_{k}^{r} S \cap H^{k-1}\right] \cdot C_{s}^{r}$. Therefore we have

$$
\operatorname{deg} S(f)=\gamma_{0}^{f} \gamma_{S}^{0}+\cdots+\gamma_{s}^{f} \gamma_{S}^{s}+\cdots+\gamma_{r}^{f} \gamma_{S}^{r}
$$

Remark 14. Theorem 13 can be used for computing $\gamma_{s}^{f}$ for a given differential equation $f$, by using a standard set of subvarieties whose cuspidal numbers are known (or easily obtainable). This method is used in the examples of section 4 .

In the smooth case we get the following

Corollary 15. Let $f$ be a differential equation on $\mathbb{P}^{n}$ and let $S$ be a smooth (or just normal) subvariety of $\mathbb{P}^{n}$ of dimension $k$. Suppose that $S(f)$ is a proper subset of $S$. Then

$$
\operatorname{deg} S(f)=\gamma_{0}^{f} \gamma_{S}^{0}+\gamma_{1}^{f} \gamma_{S}^{1}
$$

Let us work out the values of $\gamma_{S}^{0}$ and $\gamma_{S}^{1}$ for a smooth $S$. In the case $k=1, S \subseteq \mathbb{P}^{n}$ is a smooth curve. If $d$ is its degree and $g$ is genus, then $\gamma_{S}^{0}=d$ and $\gamma_{S}^{1}=2 g-2+2 d$. Note that when $n=2$, i.e. $S \subseteq \mathbb{P}^{2}$ is a smooth plane curve, the class of $S$ is $\gamma_{S}^{1}=d(d-1)$, which can be obtained by using the adjunction formula $2 g-2=d(d-3)$.

For $k>1$, let $S \subseteq \mathbb{P}^{n}$ be a smooth (or just normal) subvariety of degree $d$ and let $g$ be the genus of the generic section $C=S \cap H^{k-1}$ (which is a smooth curve). Then the degree of $C$ is $d$ and its class is $\gamma_{C}^{1}=2 g-2+2 d$. Now it is easy to see that the class of $S$ equals that of $C, \gamma_{S}^{1}=\gamma_{C}^{1}$ (for instance take a reference $x_{i}, y_{j}$ such that the $H^{k-1}$ has equations $y_{1}=0, \ldots, y_{n-k}=0, x_{1}=0$ and use lemma 12$)$.

Corollary 16. Let $f$ be a differential equation on $\mathbb{P}^{n}$ and let $S \subseteq \mathbb{P}^{n}$ be a smooth (or just normal) subvariety of degree $d$ whose generic section $S \cap H^{k-1}$ has genus $g$. Suppose that $S(f)$ is a proper subset of $S$. Then $\operatorname{deg} S(f)=\gamma_{0}^{f} d+\gamma_{1}^{f}(2 g-2+2 d)$. Furthermore, if $S$ is a hypersurface, then the formula is reduced to $\operatorname{deg} S(f)=\gamma_{0}^{f} d+\gamma_{1}^{f} d(d-1)$.

\section{EXAmples}

Theorem 13 can be used in two directions, either to compute the degree of the divisor of solutions of a differential equations on a subvariety of $\mathbb{P}^{n}$ or to extract information about a differential equation $f$ and a subvariety $S \subseteq \mathbb{P}^{n}$ once we have computed the degree of the divisor of solutions of $f$ on $S$.

Computation of $\operatorname{deg} S(f)$. Given a differential equation $f$ and a $k$-dimensional subvariety $S \subseteq \mathbb{P}^{n}$, to find the divisor $S(f)$ we proceed as follows. Take a reference $x_{i}, y_{j}$. When $S$ is smooth we find explicitly $U^{r}(f) \cap D_{k}^{r} S$ by considering the ideal generated by the equations 
defining $S$, their formal derivatives up to order $r$ together with the equation $f=0$. If $S$ is non-smooth we have to be a little bit more careful, as $D_{k}^{r} S$ is defined as the closure of $D_{k}^{r} S_{\text {reg }}$, and hence it may be smaller than the set defined by the above ideal (see example 18).

Using different references $x_{i}, y_{j}$, we find with this method all the points in $D_{k}^{r} S(f)$ not lying in

$$
\left(C_{2}^{r} \cup \ldots \cup C_{r}^{r}\right) \cap D_{k}^{r} \mathbb{P}^{n}(f) \cap D_{k}^{r} S
$$

i.e. $\left(D_{k}^{r} S(f)\right)_{\mathrm{nc}}=D_{k}^{r} S(f)-\bigcup_{s=2}^{r} C_{s}^{r}$. When $S$ is smooth $(7)$ is empty and hence $D_{k}^{r} S(f)=$ $\left(D_{k}^{r} S(f)\right)_{\mathrm{nc}}$. In general, deg $S(f)$ equals the degree of the closure of $\left(D_{k}^{r} S(f)\right)_{\mathrm{nc}}$ unless $(7)$ has $(k-1)$-dimensional components (we expect it to be $(k-2)$-dimensional).

Note that to compute $\operatorname{deg} S(f)$ we have to take a linear section $H^{k-1}$ of $S(f)$. Therefore we look for the number of solutions of the differential equation in a general $H^{k-1}$ section of $S$, which is a curve.

Example 17. Let $n=2$ and $k=1$, i.e. the case of curves in $\mathbb{P}^{2}$. Consider the smooth cubic $S$ given by $x^{3}+y^{2}=1$. To compute $\operatorname{deg} S\left(y^{\prime}\right)$, where $y^{\prime}=\frac{\partial y}{\partial x}$, we compute the number of points in

$$
\left\{\begin{array}{l}
x^{3}+y^{2}=1 \\
3 x^{2}+2 y y^{\prime}=0 \\
y^{\prime}=0
\end{array}\right.
$$

which is 4 . Now $S$ passes through the point $P=(0, \infty)$ at infinity $C^{0}$ and is tangent to $C^{0}$ there. Therefore this point counts with multiplicity 2 (otherwise compute in a different reference). So $\operatorname{deg} S\left(y^{\prime}\right)=4+2=6$.

Now let us compute $\operatorname{deg} S\left(y^{\prime \prime}\right)$, where $y^{\prime \prime}=\frac{\partial^{2} y}{\partial x^{2}}$. The number of points in

$$
\left\{\begin{array}{l}
x^{3}+y^{2}=1 \\
3 x^{2}+2 y y^{\prime}=0 \\
6 x+2\left(y^{\prime}\right)^{2}+2 y y^{\prime \prime}=0 \\
y^{\prime \prime}=0
\end{array}\right.
$$

is 8 . The point $P$ at infinity counts once, since it is a simple flex of $S$. Hence $\operatorname{deg} S\left(y^{\prime \prime}\right)=$ $8+1=9$.

Example 18. Now consider the singular cubic $T$ given by $x^{3}+y^{2}=0$. Now the closure of $D_{1}^{1} T_{\text {reg }}$ is the irreducible component of

$$
\left\{\begin{array}{l}
x^{3}+y^{2}=0 \\
3 x^{2}+2 y y^{\prime}=0
\end{array}\right.
$$

given by the equations $x=\frac{2}{3}\left(y^{\prime}\right)^{2}, y=-\frac{2}{3}\left(y^{\prime}\right)^{3}$. Hence $U^{0} \cap T\left(y^{\prime}\right)$ consists of 1 point. Also $T$ passes through $P=(0, \infty)$ at infinity with multiplicity 2 , so $\operatorname{deg} T\left(y^{\prime}\right)=1+2=3$. 
For computing $\operatorname{deg} T\left(y^{\prime \prime}\right)$ we study

$$
\left\{\begin{array}{l}
3 x-2\left(y^{\prime}\right)^{2}=0 \\
3 y+2\left(y^{\prime}\right)^{3}=0 \\
3-4 y^{\prime} y^{\prime \prime}=0 \\
3 y^{\prime}+6\left(y^{\prime}\right)^{2} y^{\prime \prime}=0 \\
y^{\prime \prime}=0
\end{array}\right.
$$

which is empty. Counting the point at infinity, $\operatorname{deg} T\left(y^{\prime \prime}\right)=1$.

Computation of $\gamma_{s}^{f}$. Apart from proposition 7 (and in some cases the extension given in remark 9), we can compute $\gamma_{s}^{f}$ by coupling $f$ with some simple examples of varieties $S \subseteq \mathbb{P}^{n}$. Many differential equations $f$ have a geometrical meaning. For instance for curves in the plane, $y^{\prime}=0$ gives the points of a curve with tangent parallel to the $x$-axis, and $y^{\prime \prime}=0$ gives the flexes of a curve. Let us work out these two examples.

For $f=y^{\prime}$, we have $\gamma_{1}^{y^{\prime}}=1$. Let $C \subseteq \mathbb{P}^{2}$ be a smooth conic, which has degree $\gamma_{C}^{0}=2$ and class $\gamma_{C}^{1}=2$. Hence $2=\operatorname{deg} C\left(y^{\prime}\right)=2 \gamma_{0}^{y^{\prime}}+2$, so $\gamma_{0}^{y^{\prime}}=0$.

For $f=y^{\prime \prime}$, proposition 7 says that $\gamma_{2}^{y^{\prime \prime}}=1$. A conic has no flexes, so $0=\operatorname{deg} C\left(y^{\prime \prime}\right)=$ $2 \gamma_{0}^{y^{\prime \prime}}+2 \gamma_{1}^{y^{\prime \prime}}$. The smooth cubic $S$ of example 17 gives first $\gamma_{1}^{S}=\operatorname{deg} S\left(y^{\prime}\right)=6$ and then $9=\operatorname{deg} S\left(y^{\prime \prime}\right)=3 \gamma_{0}^{y^{\prime \prime}}+6 \gamma_{1}^{y^{\prime \prime}}$. Therefore $\gamma_{0}^{y^{\prime \prime}}=-3$ and $\gamma_{1}^{y^{\prime \prime}}=3$.

Computation of $\gamma_{S}^{s}$. For a smooth (or normal) variety $S \subseteq \mathbb{P}^{n}$, we have that $\gamma_{S}^{0}$ is its degree and $\gamma_{S}^{1}$ its class. This can be computed by taking a general section $C=H^{k-1} \cap S$, which is a curve of degree $\gamma_{C}^{0}=\gamma_{S}^{0}$ and class $\gamma_{C}^{1}=\gamma_{S}^{1}$. So $\gamma_{S}^{1}=2 g-2+2 d$, where $g$ is the genus of $C$ and $d$ its degree. Also $\gamma_{S}^{s}=0$ for $s \geq 2$.

In the non-smooth case, things are a little bit more complicated. For instance, for the smooth cubic $S$ of example $17, \gamma_{S}^{0}=3, \gamma_{S}^{1}=6$ and $\gamma_{S}^{2}=0$. Instead for the non-smooth cubic $T$ of example 18, $\gamma_{T}^{0}=3, \gamma_{T}^{1}=\operatorname{deg} T\left(y^{\prime}\right)=3$ and $\gamma_{T}^{2}=\operatorname{deg} T\left(y^{\prime \prime}\right)-\gamma_{0}^{y^{\prime \prime}} 3-\gamma_{1}^{y^{\prime \prime}} 3=1$.

Degree of the divisor of parabolic points. As an application, we shall determine the degree of the divisor of parabolic points of a subvariety $S \subseteq \mathbb{P}^{n}$. Suppose first that $k=n-1$, i.e. $S$ is a hypersurface of $\mathbb{P}^{n}$. Parabolic points are those points of $S$ with higher contact with the tangent space than expected. In terms of a reference $x_{1}, \ldots, x_{n-1}, y$, they are the solutions to the differential equation

$$
f=\operatorname{det}\left(\frac{\partial^{2} y}{\partial x_{i} \partial x_{j}}\right)
$$

To compute the invariants of $f$ we work as follows. By remark $9, \gamma_{2}^{f}=n-1$. Now for a smooth quadric $C \subseteq \mathbb{P}^{n}$ there are no parabolic points, so $0=\operatorname{deg} C(f)=2 \gamma_{0}^{f}+2 \gamma_{1}^{f}$, and $\gamma_{1}^{f}=-\gamma_{0}^{f}$. Now let $S \subseteq \mathbb{P}^{n}$ be the smooth cubic given by $x_{1}^{3}+\cdots+x_{n-1}^{3}+y^{2}+1=0$. It is easy to compute

$$
\begin{cases}\frac{\partial^{2} y}{\partial x_{i}^{2}}=-\frac{3 x_{i}}{y}-\frac{9 x_{i}^{4}}{4 y^{3}}, & 1 \leq i \leq n-1 \\ \frac{\partial^{2} y}{\partial x_{i} \partial x_{j}}=\frac{-9 x_{i}^{2} x_{j}^{2}}{4 y^{3}}, & 1 \leq i, j \leq n-1, i \neq j\end{cases}
$$


so that $\operatorname{det}\left(\frac{\partial^{2} y}{\partial x_{i} \partial x_{j}}\right)=(-3)^{n-1} x_{1} \cdots x_{n-1}\left(y^{2}-3\right) / 4 y^{n+1}$ and hence $3(n+1)=\operatorname{deg} S(f)=$ $3 \gamma_{0}^{f}+6 \gamma_{1}^{f}$. This yields $\gamma_{1}^{f}=-\gamma_{0}^{f}=n+1$. Our conclusion is that for a hypersurface $S \subseteq \mathbb{P}^{n}$, the degree of the divisor of parabolic points is

$$
\operatorname{deg} S(f)=-(n+1) \gamma_{S}^{0}+(n+1) \gamma_{S}^{1}+(n-1) \gamma_{S}^{2} .
$$

If $S$ is a smooth hypersurface of degree $d$, it reduces to

$$
\operatorname{deg} S(f)=-(n+1) d+(n+1) d(d-1)=(n+1) d(d-2) .
$$

This agrees with the following alternative argument (only applies to the smooth case): if $S$ is given by the equation $F\left(x_{0}, \ldots, x_{n}\right)=0$, then the parabolic points are the intersection of $F=0$ and $\operatorname{det}\left(\frac{\partial^{2} F}{\partial X_{i} \partial X_{j}}\right)=0$, and so form a divisor of degree $(n+1) d(d-2)$.

For the general case $0<k<n$, we consider a reference $x_{i}, y_{j}$ and fix $1 \leq r \leq n-k$. Then the parabolic points in the $y_{r}$-direction are the solutions to the differential equation

$$
f_{r}=\operatorname{det}\left(\frac{\partial^{2} y_{r}}{\partial x_{i} \partial x_{j}}\right) \text {. }
$$

Working as above we have the formula

$$
\operatorname{deg} S\left(f_{r}\right)=-(n+1) \gamma_{S}^{0}+(n+1) \gamma_{S}^{1}+(n-1) \gamma_{S}^{2} .
$$

\section{The ReAl CASE}

In this section, our purpose is to extend theorem 13 to the case of real projective varieties inside the real projective space $\mathbb{P}_{\mathbb{R}}^{n}$. We are not going to develop the general theory of data schemes for smooth real algebraic varieties, but only to outline the construction of $D_{k}^{r} \mathbb{P}_{\mathbb{R}}^{n}$.

First of all fix a trivialisation on $\mathbb{P}^{n}$ as in section 2 , so $\mathbb{P}^{n}=\mathbb{P}\left(\mathbb{C}^{n+1}\right)$. Again $1 \leq k \leq$ $n-1$. There is an anti-holomorphic involution $\sigma: \mathbb{P}^{n} \rightarrow \mathbb{P}^{n}$ coming from conjugation on $\mathbb{C}^{n+1}$, whose fixed point set is $\mathbb{P}_{\mathbb{R}}^{n}$. Inductively, $\sigma$ induces anti-holomorphic involutions $\sigma_{r}: D_{k}^{r} \mathbb{P}^{n} \rightarrow D_{k}^{r} \mathbb{P}^{n}, r \geq 1$. We define $D_{k}^{r} \mathbb{P}_{\mathbb{R}}^{n}$ to be the fixed point set of $\sigma_{r}$. The maps $b_{r, s}$ of (5) restrict to $D_{k}^{r} \mathbb{P}_{\mathbb{R}}^{n}$ and so there are well-defined maps $b_{r, s}: D_{k}^{r} \mathbb{P}_{\mathbb{R}}^{n} \rightarrow D_{k}^{s} \mathbb{P}_{\mathbb{R}}^{n}$. It is easy to see that we can construct $D_{k}^{r} \mathbb{P}_{\mathbb{R}}^{n}$ as follows. We take $D_{k}^{0} \mathbb{P}_{\mathbb{R}}^{n}=\mathbb{P}_{\mathbb{R}}^{n}, D_{k}^{1} \mathbb{P}_{\mathbb{R}}^{n}=\mathrm{Gr}_{k} T \mathbb{P}_{\mathbb{R}}^{n}$, the real Grassmannian of $k$-planes in the tangent bundle $T \mathbb{P}_{\mathbb{R}}^{n}$, and for $r \geq 2, D_{k}^{r} \mathbb{P}_{\mathbb{R}}^{n}=\operatorname{Gr}_{k} \mathcal{F}_{r-1}^{\mathbb{R}}$, where the real (Semple) bundle $\mathcal{F}_{r-1}^{\mathbb{R}}$ is defined by a diagram as (1), where the relative tangent bundles and the universal sequence of the right hand side are understood to be real. These $D_{k}^{r} \mathbb{P}_{\mathbb{R}}^{n}$ are also smooth compact differentiable manifolds.

The involution $\sigma_{r}$ takes $C^{r}$ to itself and the fixed point set will be called $C_{\mathbb{R}}^{r}$. This is a smooth 1-codimensional real algebraic subvariety of $D_{k}^{r} \mathbb{P}_{\mathbb{R}}^{n}$. Clearly $C_{\mathbb{R}}^{0} \subseteq \mathbb{P}_{\mathbb{R}}^{n}$ is the hyperplane. The other $C_{\mathbb{R}}^{r}$ can be also defined as the cuspidal locus of $D_{k}^{r} \mathbb{P}_{\mathbb{R}}^{n}$, namely the Schubert special cycle of real $k$-planes of $\mathcal{F}_{r-1}^{\mathbb{R}}$ meeting $T D_{k}^{r-1} \mathbb{P}_{\mathbb{R}}^{n} / D_{k}^{r-2} \mathbb{P}_{\mathbb{R}}^{n}$. Again $C_{\mathbb{R}, s}^{r}$ are defined either as the fixed point set of $\sigma_{r}$ on $C_{s}^{r}$ or as $b_{r, s}^{-1}\left(C_{\mathbb{R}}^{s}\right)$.

We can parallel the discussion in section 2 to see that

$$
U_{\mathbb{R}}^{r}=D_{k}^{r} \mathbb{P}_{\mathbb{R}}^{n} \backslash\left(C_{\mathbb{R}, 0}^{r} \cup \ldots \cup C_{\mathbb{R}, s}^{r} \cup \ldots \cup C_{\mathbb{R}, r}^{r}\right)
$$


are cartesian powers of $\mathbb{R}$. Indeed, $U_{\mathbb{R}}^{0}=\mathbb{R}^{k} \times \mathbb{R}^{n-k}$ has real coordinates (the restriction to $U_{\mathbb{R}}^{0} \subseteq U^{0}$ of) $x_{1}, \ldots, x_{k}, y_{1}, \ldots, y_{n-k}$. In general

$$
U_{\mathbb{R}}^{r} \cong U_{\mathbb{R}}^{r-1} \times V_{\mathbb{R}}^{r}
$$

where $V_{\mathbb{R}}^{r}=\operatorname{Hom}\left(\left(\mathbb{R}^{k}\right)^{\otimes r}, \mathbb{R}^{n-k}\right)$, for any $r \geq 1$. Alternatively, $\sigma_{r}$ restricts to $U^{r}$ and the fixed point locus is $U_{\mathbb{R}}^{r}$. The coordinates for $U_{\mathbb{R}}^{r}$ will be

$$
x_{1}, \ldots, x_{k}, y_{1}, \ldots, y_{n-k} \quad \text { and } \quad y_{i_{1}, \ldots, i_{s}}^{j}=\frac{\partial^{s} y_{j}}{\partial x_{i_{s}} \cdots \partial x_{i_{1}}}
$$

for any $1 \leq s \leq r, 1 \leq j \leq n-k$, and $1 \leq i_{1}, \ldots, i_{s} \leq k$.

Lemma 19. $C_{0, \mathbb{R}}^{r}, \ldots, C_{s, \mathbb{R}}^{r}, \ldots, C_{r, \mathbb{R}}^{r}$ form a basis for $H^{1}\left(D_{k}^{r} \mathbb{P}_{\mathbb{R}}^{n} ; \mathbb{Z} / 2 \mathbb{Z}\right)$, where we name equally the algebraic subvarieties and the cohomology classes they represent through Poincaré duality.

Proof. Given the description of the real Grassmannian as a homogeneous space

$$
\mathrm{Gr}_{k} \mathbb{R}^{n}=O(n) / O(k) \times O(n-k),
$$

it is easy to prove that the fundamental group $\pi_{1}\left(\mathrm{Gr}_{k} \mathbb{R}^{n}\right)=\mathbb{Z} / 2 \mathbb{Z}$ for $n>2$ and $\pi_{1}\left(\mathrm{Gr}_{1} \mathbb{R}^{2}\right)=$ $\pi_{1}\left(\mathbb{R} \mathbb{P}^{1}\right)=\mathbb{Z}$. So $H^{1}\left(\mathrm{Gr}_{k} \mathbb{R}^{n} ; \mathbb{Z} / 2 \mathbb{Z}\right)=\mathbb{Z} / 2 \mathbb{Z}$ and it is generated by the cuspidal subvariety. The Serre spectral sequence of the fibration

$$
\mathrm{Gr}_{k} \mathbb{R}^{\bullet} \rightarrow D_{k}^{r} \mathbb{P}_{\mathbb{R}}^{n}=\mathrm{Gr}_{k} \mathcal{F}_{r-1}^{\mathbb{R}} \rightarrow D_{k}^{r-1} \mathbb{P}_{\mathbb{R}}^{n}
$$

implies that $H^{1}\left(D_{k}^{r} \mathbb{P}_{\mathbb{R}}^{n} ; \mathbb{Z} / 2 \mathbb{Z}\right)$ is generated by $C_{0, \mathbb{R}}^{r}, \ldots, C_{r, \mathbb{R}}^{r}$. Now the 1 -cycles $c_{s, \mathbb{R}}^{r}$ are defined as in the proof of proposition 7 (alternatively as the fixed locus of $\sigma_{r}$ on $c_{s}^{r}$ ) and the homology classes they represent in $H_{1}\left(D_{k}^{r} \mathbb{P}_{\mathbb{R}}^{n} ; \mathbb{Z} / 2 \mathbb{Z}\right)$ satisfy $c_{t, \mathbb{R}}^{r} \cdot C_{s, \mathbb{R}}^{r}=0$, for $t<s$ and $c_{s, \mathbb{R}}^{r} \cdot C_{s, \mathbb{R}}^{r}=1(\bmod 2)$. This shows that $C_{s, \mathbb{R}}^{r}$ are linearly independent.

Recall that the cohomology ring of $\mathbb{P}_{\mathbb{R}}^{n}$ (with $\mathbb{Z} / 2 \mathbb{Z}$-coefficients) is $(\mathbb{Z} / 2 \mathbb{Z})[H] / H^{n+1}$, where $H$ stands for the hyperplane class. Again $H=C_{0}^{r}=b_{r, 0}^{-1}(H)$ is the hyperplane in $D_{k}^{r} \mathbb{P}^{n}$. Now we are in the position of defining differential equations on $\mathbb{P}_{\mathbb{R}}^{n}$.

Definition 20. A differential equation on $\mathbb{P}_{\mathbb{R}}^{n}$ relative to a reference $x_{i}, y_{j}$ is a non-zero algebraic equation with real coefficients

$$
f\left(x_{i}, y_{j}, y_{i}^{j}, y_{i_{1}, i_{2}}^{j}, \cdots, y_{i_{1}, \ldots, i_{r}}^{j}\right)=0,
$$

on $U_{\mathbb{R}}^{r}$, for some $r \geq 1$.

This time the zero locus of such $f$ is not necessarily a hypersurface of $D_{k}^{r} \mathbb{P}_{\mathbb{R}}^{n}$ (it might even be empty). But we may still define the numbers $\gamma_{s}^{f} \in \mathbb{Z} / 2 \mathbb{Z}$ by looking at the component in $H^{1}\left(D_{k}^{r} \mathbb{P}^{n} ; \mathbb{Z} / 2 \mathbb{Z}\right)$ defined by the closure of the zero locus of $f$ in $U_{\mathbb{R}}^{r} \subseteq D_{k}^{r} \mathbb{P}_{\mathbb{R}}^{n}$. So

$$
\left[D_{k}^{r} \mathbb{P}_{\mathbb{R}}^{n}(f)\right]_{1}=\gamma_{0}^{f} C_{0, \mathbb{R}}^{r}+\cdots+\gamma_{r}^{f} C_{r, \mathbb{R}}^{r}
$$

We leave the following analogue of proposition 7 to the reader.

Proposition 21. Let $f$ be a differential equation on $\mathbb{P}_{\mathbb{R}}^{n}$ relative to a reference $x_{i}, y_{j}$. 
- If $f$ is distinguished in the variable $y_{1,(r), 1}^{1}$, then $\gamma_{r}^{f}=\operatorname{deg} f\left(0, \ldots, y_{1,(r), 1}^{1}, \ldots, 0\right)$.

- If $f$ is distinguished in the variable $y_{1}^{1}$, then $\gamma_{1}^{f}=\operatorname{deg} f\left(0, \ldots, y_{1}^{1}, \ldots, 0\right)$.

- If $f$ is distinguished in the variable $x_{1}$, then $\gamma_{0}^{f}=\operatorname{deg} f\left(x_{1}, \ldots, 0\right)$.

Let $S \subseteq \mathbb{P}_{\mathbb{R}}^{n}$ be a real algebraic $k$-dimensional subvariety (this is the zero locus of polynomial equations with real coefficients such that it has a dense open subset that is a smooth differentiable manifold of dimension $k$ ). Considering the same equations in $\mathbb{P}^{n}$, we get a complex subvariety $S_{\mathbb{C}} \subseteq \mathbb{P}^{n}$ on which $\sigma$ acts with fixed point set $S$. We define $D_{k}^{r} S \subseteq D_{k}^{r} \mathbb{P}_{\mathbb{R}}^{n}$ as the fixed point set of $\sigma_{r}$ on $D_{k}^{r} S_{\mathbb{C}} \subseteq D_{k}^{r} \mathbb{P}^{n}$. There is a natural map $D_{k}^{r} S \rightarrow S$, which is an isomorphism over the smooth part. When $S$ is smooth then $S \cong D_{k}^{r} S$ and $D_{k}^{r} S$ is disjoint from the cuspidal divisors $C_{s, \mathbb{R}}^{r}, s \geq 2$. By analogy with lemma 12, we define the cuspidal numbers of $S$ as follows

Definition 22. For any $0 \leq s \leq r$, we define the $s^{t h}$-cuspidal number of $S$ as $\gamma_{S}^{s}=$ $D_{k}^{r} S \cap C_{s}^{r} \cap H^{k-1} \in \mathbb{Z} / 2 \mathbb{Z}$ (computed in the cohomology ring of $D_{k}^{r} \mathbb{P}_{\mathbb{R}}^{n}$ ).

Note also that when $S \subseteq \mathbb{P}_{\mathbb{R}}^{n}$ is a smooth differentiable $k$-dimensional manifold, $D_{k}^{r} S \subseteq \mathbb{P}_{\mathbb{R}}^{n}$ is also defined by mimicking the algebraic construction and $D_{k}^{r} S \rightarrow S$ is a diffeomorphism. In this case $\gamma_{S}^{0}$ and $\gamma_{S}^{1}$ are defined as above.

Given a differential equation $f$ on $\mathbb{P}^{n}$, we define $S(f)$ as the homology class in $H_{k-1}\left(\mathbb{P}_{\mathbb{R}}^{n} ; \mathbb{Z} / 2 \mathbb{Z}\right)$ given as the push-forward of

$$
D_{k}^{r} S(f)=D_{k}^{r} S \cap D_{k}^{r} \mathbb{P}_{\mathbb{R}}^{n}(f) \in H_{k-1}\left(D_{k}^{r} \mathbb{P}_{\mathbb{R}}^{n} ; \mathbb{Z} / 2 \mathbb{Z}\right) .
$$

Its degree is

$$
\operatorname{deg} S(f)=S(f) \cap H^{k-1} \in \mathbb{Z} / 2 \mathbb{Z}
$$

The following result is proved much in the same way as theorem 13.

Theorem 23. Let $f$ be a differential equation on $\mathbb{P}_{\mathbb{R}}^{n}$ with $\gamma_{s}^{f} \in \mathbb{Z} / 2 \mathbb{Z}, 0 \leq s \leq r$. Let $S$ be a real algebraic subvariety of $\mathbb{P}_{\mathbb{R}}^{n}$ of dimension $k$. Then

$$
\operatorname{deg} S(f)=\gamma_{0}^{f} \gamma_{S}^{0}+\cdots+\gamma_{s}^{f} \gamma_{S}^{s}+\cdots+\gamma_{r}^{f} \gamma_{S}^{r} \in \mathbb{Z} / 2 \mathbb{Z}
$$

If $S$ is smooth or $S$ is just a differentiable manifold of dimension $k$, we have

$$
\operatorname{deg} S(f)=\gamma_{0}^{f} \gamma_{S}^{0}+\gamma_{1}^{f} \gamma_{S}^{1} \in \mathbb{Z} / 2 \mathbb{Z}
$$

Umbilical points. We shall compute the parity of the degree of the subset of umbilical points for surfaces in $\mathbb{P}_{\mathbb{R}}^{3}$. For a reference $x_{1}, x_{2}, y$, these are the points where the Hessian $\left(\frac{\partial^{2} y}{\partial x_{i} \partial x_{j}}\right)$ is diagonal. Therefore we look for the solutions to

$$
f=\left(\frac{\partial^{2} y}{\partial x_{1}^{2}}-\frac{\partial^{2} y}{\partial x_{2}^{2}}\right)^{2}+4\left(\frac{\partial^{2} y}{\partial x_{1} \partial x_{2}}\right)^{2} .
$$

Clearly $\gamma_{2}^{f}=2=0$ (we work over $\mathbb{Z} / 2 \mathbb{Z}$ ). For the cubic $S$ given by $x_{1}^{3}+y^{2}+1=0$ with $\gamma_{S}^{0}=1$ and $\gamma_{S}^{1}=0$ (see example 17 ), the equation $f$ reduces to $\left(\frac{\partial^{2} y}{\partial x_{1}^{2}}\right)^{2}$, so $\gamma_{0}^{f}=$ $\operatorname{deg} S\left(\left(\frac{\partial^{2} y}{\partial x_{1}^{2}}\right)^{2}\right)=2 \operatorname{deg}\left(\frac{\partial^{2} y}{\partial x_{1}^{2}}\right)=0$. For the cubic $T$ given by $x_{1}^{3}+y^{2}=0$, with $\gamma_{S}^{0}=1$ and 
$\gamma_{S}^{1}=1$ (see example 18), the equation $f$ reduces to $\left(\frac{\partial^{2} y}{\partial x_{1}^{2}}\right)^{2}$, so $\gamma_{0}^{f}+\gamma_{1}^{f}=\operatorname{deg} S\left(\left(\frac{\partial^{2} y}{\partial x_{1}^{2}}\right)^{2}\right)=0$. Therefore $\gamma_{0}^{f}=\gamma_{1}^{f}=\gamma_{2}^{f}=0$ and the number of umbilical points of any surface $S \subseteq \mathbb{P}_{\mathbb{R}}^{3}$ (smooth or not) is always even.

\section{REFERENCES}

1. M. Belghiti, Variétés des points infinitement voisins d'ordre $n$ de points du plan, C.R. Acad. Sci. Paris, 314 Série I, 1992, 541-545.

2. S. Colley and G. Kennedy, A higher-order contact formula for plane curves, Comm. in Algebra, 19 1991, 479-508.

3. S. Colley and G. Kennedy, Triple and quadruple contact of plane curves, Proc. Zeuthen Symp. Contemp. Math. 123 1991, 31-559.

4. A. Collino, Evidence for a conjecture of Ellingsrud and Strømme on the Chow ring of $\mathrm{Hilb}_{d} \mathbb{P}^{2}$, Illinois Jour. Math., 32 1988, 171-210.

5. E. Arrondo, I. Sols and R. Speiser, Global moduli of contacts, Arkiv för matematik, 35 1997, $1-57$.

6. G. Halphen, Sur la recherche des points d'une courbe algébrique plane, qui satisfont à une condition exprimée par une équation différentielle algébrique, et sur les questions analogues dans l'espace, Oeuvres, Gauthier-Villars, Paris, 1 1916, 475-542.

7. R. Hartshorne, Algebraic geometry, Springer-Verlag, 1978.

Departamento de Álgebra, Geometría y Topología

Facultad de Ciencias

Universidad de Málaga

Campus de Teatinos, s/n

29071 Málaga

Spain

E-mail:vmunoz@agt.cie.uma.es

Departamento de Álgebra

Facultad de Ciencias Matemáticas

Universidad Complutense de Madrid

28040 Madrid

Spain

E-mail: sols@mat.ucm.es 